\title{
Finding common ground
}

\author{
James P. Evans, MD, PhD
}

It is a rare privilege to be a part of the Medical Genetics community at this moment in history. Due largely to breathtaking technological advances over the past few years, we are gaining tremendous insights into humanity itself, and we are beginning to apply this new technology and our burgeoning knowledge to the care of patients in ways never before imagined. Being in our field at this moment feels a bit like what it must have been like to have been a part of the physics community in the first part of the 20th century when new insights were coming fast and furiously, and the ground was rapidly shifting under everyone's feet.

Living in such times can be unsettling as well as exciting. Witness the furor over the American College of Medical Genetics and Genomics (ACMG) recommendations on reporting incidental findings ${ }^{1}$ that struck quite a chord in our community. These guidelines address only one facet of many ongoing debates about how to best use, apply, and approach massive amounts of genomic data. The controversy and arguments that by now most of us are (all too!) familiar with have been widely covered in the popular press and in the academic literature outside of our core journals. ${ }^{2-6}$ The vibrancy and breadth of these discussions reflect the fact that we humans are fascinated by ourselves (and therefore by our genome). But more critically and optimistically for our field, the intensity of these debates reflects the growing relevance of medical genetics to the rest of medicine and to the lives of others. Our field matters in a way that it never has before.

Here, at Genetics in Medicine, we have received an unprecedented number of contributions that address how to handle incidental findings. Some of these manuscripts directly address the ACMG recommendations, whereas others grapple in a more general way with optimal approaches to dealing with the massive amounts of incredibly heterogeneous data that we now routinely generate. Some are opinion pieces and explore (or advocate for) a specific approach, whereas others have begun to gather data relevant to the formulation of optimal policies. We are delighted that we have received manuscripts with data on different approaches to handling genomic information, as ultimately data must guide our policies. But informed opinions matter too, because our application of new technology and new knowledge ultimately hinge on values, and pure data are only part of the decision-making process in such a complex arena. Thoughtful opinions are especially important at this early stage when very few patients have actually undergone genome-scale sequencing, leaving us with only modest experience.

Because of the overwhelming number of submissions we have received that focus in some way on incidental findings, we felt that a theme issue was appropriate and might facilitate progress in forging solutions to our community's challenges. I am particularly pleased that GIM can serve as a forum for the discussion of these critical (albeit contentious) issues. Throughout this issue, you will find a range of opinions and data that might begin to inform standard approaches to handling heterogeneous genomic data in the clinical setting. Reading through the various articles will hopefully help you begin to make up your mind about how we should move forward and how we can settle our differences.

And we must move forward. These questions are too important-and our field is now too relevant to both patients and the public-for us to allow ourselves to get caught in a cul-desac of endless academic argument and debate. It is abundantly clear to me that we are not going to all think or act as a single mind on these issues anytime soon, since, after all, our positions are strongly influenced by (necessarily variable) personal values. With the brilliant arguments that have been put forth by both the sides, if we were going to convince everyone else that our particular position is "the right way" to handle incidental findings, we would have arrived at that point already (I hate to invoke "sides" here, but that is the reality).

I have a simple request to everyone who reads these articlesthink hard about your own position-and think just as hard about where you could compromise with those who have a different opinion. After all, the simple truth is that none of us know for sure what the best policy is. That will take much time and the generation of much data (something we must commit to doing). So, what could you live with that is being advocated by the "other side"? Real compromise is excruciatingly uncomfortable (actually, if it is not uncomfortable, then it is probably not much of a compromise). But I see compromise as the only way forward and an eminently sensible way forward when, again, the truth is that none of us know for sure what the best course is.

In that spirit of compromise, I will tell you where I personally come down, having read (way too many) positions on this matter, listened to strong (but thankfully, largely polite) debate, and obsessed (way too much) about it. I like the ACMG recommendations. As a practicing internist, I feel that they bring

${ }^{1}$ Department of Genetics, University of North Carolina at Chapel Hill, Chapel Hill, North Carolina, USA. Correspondence: James P. Evans (jpevans@med.unc.edu) 
genomics in line with the way medicine is currently practiced. I am all for patient autonomy but also recognize that affording autonomy is not always simple in the clinical world and that "patient choice" is fraught, complex, at times unrealizable and must be constrained by professional judgment. ${ }^{7}$ But I also will readily admit that while I feel comfortable with my formulation of the issue, I might be wrong (imagine that). I would add that it should give all of us pause to see the numerous thoughtful, intelligent, and eminently qualified colleagues in our community who have lined up on the other side of whatever position we may have taken.

So, for whatever it is worth, here is where I can compromise: let us routinely examine the "ACMG 56" for clearly deleterious mutations when genome-scale sequencing is performed. But let us allow patients to opt out of such analysis of their specimen. I am uncomfortable with this for some reasons, mostly because I worry that this affords patients an illusory degree of autonomy and that in reality, discussions about an unlikely and hypothetical scenario that they choose to opt out of does not really boil down to an informed choice. And I worry that such an illusory choice will come back to haunt them with highly problematic consequences. But, again, that is the nature of compromise. If you do not feel that you are giving up something important then you have not probably compromised. And, after all, maybe I am wrong (it has certainly happened before). I'll be the first to admit that those who identify patient autonomy as a critical feature of this debate make some compelling arguments. And make no mistake; setting opt-out as the default also demands compromise by others. After all, it means that we usually will examine the "ACMG 56 " for deleterious mutations since I suspect that most patients will probably not exercise an opt-out option. Therefore, there are those who will be uncomfortable looking for these mutations as the default procedure. But, again, discomfort by both parties is part of any compromise. The twin realities are that none of us know for sure what to do at this critical juncture in Medical Genetics but that there exists a real and immediate need to formulate policies to guide laboratories, clinicians, and patients as we begin to implement genomic medicine. Finding (perhaps uncomfortable) common ground amid the strong arguments from both sides seems the only way forward until we have more data.

Which brings me to my final point, and back to this special themed issue of Genetics in Medicine. We need more data. Not just about how people (both providers and patients) feel regarding how to strike the appropriate balance regarding the return of results, but data about what patients actually choose when given the opportunity of finding out such things. We need to study whether patients actually understand what they are opting in or out of and whether they regret their choice later, along with a whole host of other ethical, legal, and social implications (ELSI) questions. And we need hard-core, unbiased genetic data that we could only have dreamed about acquiring a few years ago but are now finally poised to collect. For example, what is the real penetrance of deleterious mutations in the ACMG 56 (a list which encompasses some of the most studied genes in the history of genetics)? The answer to this fundamental question bears critically upon whether we should report such mutations when collaterally generated in the course of genome-scale sequencing and also upon whether we should contemplate going out and looking for such mutations in the general population as a public health effort. ${ }^{8}$

Let's find common ground and move ahead together.

\section{ACKNOWLEDGMENTS}

The views expressed here are solely those of the author. They are not meant to reflect any official view of the American College of Medical Genetics and Genomics or the Board of ACMG.

\section{DISCLOSURE}

The author declares no conflict of interest.

\section{REFERENCES}

1. Green RC, Berg JS, Grody WW, et al. ACMG recommendations for reporting of incidental findings in clinical exome and genome sequencing. Genet Med 2013;15:565-574.

2. McGuire $A L$, Joffe $S$, Koenig $B A$, et al. Ethics and genomic incidental findings. Science 2013;340:1047-1048.

3. Klitzman R, Appelbaum PS, Chung W. Return of secondary genomic findings vs patient autonomy: implications for medical care. JAMA 2013;310:369-370.

4. Wolf SM, Annas GJ, Elias S. Point-counterpoint. Patient autonomy and incidental findings in clinical genomics. Science 2013;340:1049-1050.

5. Green RC, Lupski JR, Biesecker LG. Reporting genomic sequencing results to ordering clinicians: incidental, but not exceptional. JAMA 2013;310:365-366.

6. Ross LF, Rothstein MA, Clayton EW. Mandatory extended searches in all genome sequencing: "incidental findings," patient autonomy, and shared decision making. JAMA 2013;310:367-368.

7. McGuire AL, McCullough LB, Evans JP. The indispensable role of professional judgment in genomic medicine. JAMA 2013;309:1465-1466.

8. Evans JP, Berg JS, Olshan AF, Magnuson T, Rimer BK. We screen newborns, don't we?: realizing the promise of public health genomics. Genet Med 2013;15:332-334.e 